\title{
Vehicle Handling Dynamics State Estimation Based on Strong Tracking Filter
}

\author{
Shu-en Zhao, Yu-ling Li and Xian Qu \\ College of Mechantronics and Automobile Engineering, Chongqing JiaoTong \\ University, Chongqing, China \\ zse0916@163.com
}

\begin{abstract}
Due to some key state parameters of vehicle handling stability control are difficult to measure directly, the state optimization estimation algorithm of multi-sensor linear combination based on Strong Tracking Filter (STF) was proposed. Four degrees of freedom vehicle nonlinear dynamics model including longitudinal, lateral and roll motion were established. With the estimator of multi-sensors information fusion and the STF theory, the vehicle handling dynamics states estimation were simulated and analyzed. The result shows that the STF offers higher performance potential. Not only does it solve the problems of the state estimation value deviating from the true system states due to the model uncertainty, but also can inhibit the filtering divergence effectively. The technology of state estimation with the STF has wide range of adaptive tracking capability. It provides a real-time, accurate and low cost soft-sensing technology for vehicle advance control.
\end{abstract}

Keywords: Vehicle Engineering, State Estimation, Information Fusion, Strong Tracking Filtering

\section{Introduction}

With the development of vehicle chassis advanced control techniques, the performance of vehicle handling stability, safety and riding comfort have improved greatly. These advanced controllers based on multi-variable control methods need accurate information about vehicle states, such as sideslip angle, sideslip velocity, yaw rate, roll angle and roll rate. However, not all of the vehicles states can be measured directly, such as sideslip velocity. Besides, the costs are expensive for direct measure, such as yaw and roll rates. Meanwhile, because of the sensors have calibrated and drift error, the measurement noise cannot confirm its distribution character, which brings a lot of difficulties for vehicle handling state information measure precisely. So, using the multi-sensors measurement information to achieve the vehicle real-time state information and judge the vehicle running attitude is very important for the vehicle active safety, handling stability and riding comfort control. It is also the prerequisite to the vehicle close-loop feedback control.

There are many studies on the vehicle state estimation with many methods and objectives [1-4]. Wenzel, Thomas A. estimated the vehicle state and parameter with dual Extended Kalman Filter (EKF) [5-6]. M. C. Best, T. J. Gordon and Medy Satria estimated the vehicle states by Kalman or EKF [7]. Hyeongcheol Lee applied reliability indexed sensor fusion to estimate the vehicle velocity [8]. Rezaeian, A. et al., proposed a modelbased "Cascaded Dual Extended Kalman Filter" (CDEKF) for combined vehicle state estimation, namely, tire vertical forces and parameter identification [9]. Gabriel Agamennoni, et al., presented a robust non-linear smoothing algorithm for vehicle state estimation. This algorithm is extremely robust to outliers and missing data. It also can 
handle state-dependent noise effectively [10]. Alexander Katriniok, et al. proposed a model-based predictive control approach for vehicle guidance combining longitudinal and lateral. The controller has been designed for an automotive collision avoidance system which aims at a desired evasion trajectory at the handling limits [11]. Jin Zhao, et al. proposed a vehicle lateral state observer which used the Kalman-Bucy filter for the vehicle lateral velocity and yaw rate estimation [12]. Due to the uncertainty of modeling dynamic system and the instability of the component, it brings a lot of difficulties in giving a statistical property description of the system and observing noise in practical application. So, the state estimation results of the conventional Kalman or EKF with predefined noise are not very accurate.

In this paper, according with the 4-DOF freedom vehicle dynamic model, the simple method is implemented to estimate the vehicle states of yaw rate, roll angle velocity, roll angle and side-slip angle based on Strong Tracking Filter (STF) theory and multi-sensor data fusion [13-14]. The simulation results indicate that STF can improve the reliability of the vehicle state estimation system and the accuracy of estimates better than EKF.

\section{Vehicle Nonlinear State Estimation Procedure}

\subsection{Vehicle Nonlinear State Estimation Principle}

In principle, the vehicle handling dynamics state of yaw rate and side slip angle can be estimated by using kinematic relationships, which is from the measured speed of undriven wheels or is from measured lateral acceleration. However, these estimates are reasonably good in restrictive conditions.

Another way to estimate the vehicle yaw rate and side slip angle uses a speeddependent dynamic model of vehicle motion in the yaw plane with steering angle as input. In this approach, the estimates tend to deviate from the actual values as a result of mismatch between the vehicle actual parameters and those used by the model, or the disturbances, such as lateral forces and moments due to side wind. In order to minimize these effects, the model must include the feedback of the error signals, that is, the differences between the measured signals and that predicted by the model. The basic block diagram of vehicle handling dynamics state estimation is shown in Figure 1.

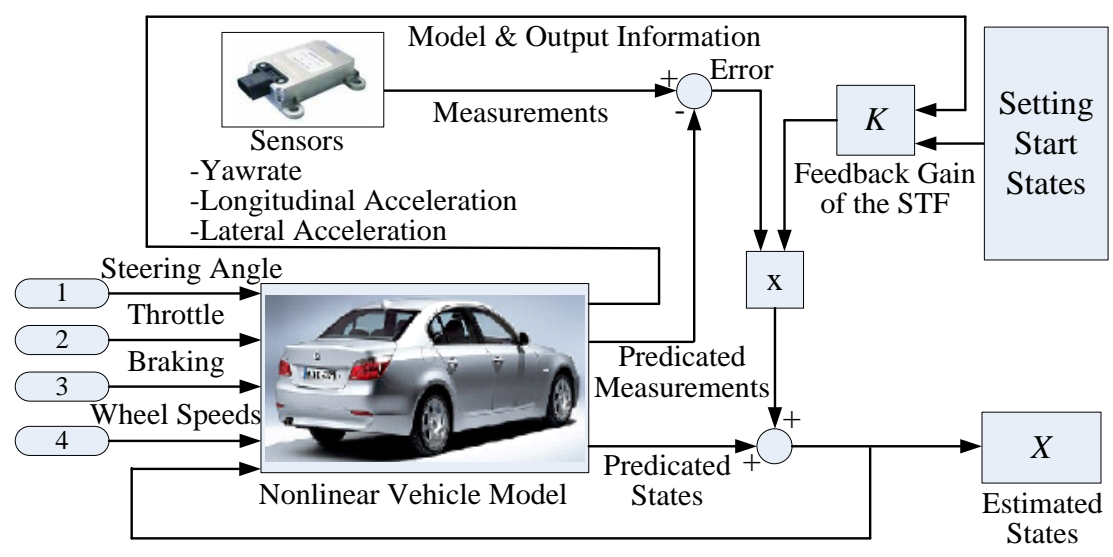

Figure 1. Vehicle State Estimation Flowchart

\subsection{Strong Tracking Filter Design}

Strong Tracking Filter adopts the orthogonal theory based on EKF and introduces a suboptimal fading coefficient 4 [9]. The force residual error is orthogonal or rough orthogonal. So, STF has strong ability to track the status of mutation through covariance matrix, the corresponding gain matrix and the real-time states predict error. It makes the 
measurement errors orthogonal approximatively through real-time gain adjustment. The target tracking performance is improved by STF.

For a discrete time nonlinear system, there are equations as following

$\left\{\begin{array}{l}x(k+1)=f_{d}(k, u(k), x(k))+\Gamma(k) w(k) \\ y(k+1)=h_{d}(k+1, x(k+1))+v(k+1)\end{array}\right.$

Where, $x$ is state, $u$ input, $y$ output and $x \in R^{n}, u \in R^{p}, y \in R^{m}$; the nonlinear function $f_{d}: R^{p} \times R^{n} \rightarrow R^{n}$ and $h_{d}: R^{n} \rightarrow R^{m}$ have continuous partial derivative for state $x$; the process noise $w(k) \in R^{q}$ is a white noise with zero mean and variance $Q(k)$; noise input matrix $\Gamma(k)$ used to describe how to allocate $w(k)$ to each state.

The STF process with a sampling period of nonlinear system is shown in Figure 2.

\begin{tabular}{|c|c|}
\hline Time update (Predict) & Measurement update (Correct) \\
\hline $\begin{array}{l}\text { State predict equation } \\
\hat{x}(k+1 \mid k)=f_{d}(k, u(k), \hat{x}(k \mid k)) \\
\text { Error covariance predict equation } \\
P(k+1 \mid k)=\Lambda(k+1) F(k, u(k), \hat{x}(k \mid k)) \\
\cdot P(k \mid k) F^{\mathrm{T}}(k, u(k), \hat{x}(k \mid k)) \\
+\Gamma(k) Q(k) \Gamma^{\mathrm{T}}(k) \\
\text { Residual sequence } \\
\gamma(k+1)=y(k+1)-h_{d}(k+1, \hat{x}(k+1 \mid k))\end{array}$ & $\begin{array}{l}\text { Gain equation } \\
K(k+1)=P(k+1 \mid k) H^{\mathrm{T}}(k+1, \hat{x}(k+1 \mid k)) \\
\cdot[H(k+1, \hat{x}(k+1 \mid k)) P(k+1 \mid k) \\
\left.\cdot H^{\mathrm{T}}(k+1, \hat{x}(k+1 \mid k))+R(k+1)\right]^{-1} \\
\text { Filter equation } \\
\hat{x}(k+1 \mid k+1)=\hat{x}(k+1 \mid k)+K(k+1) \gamma(k+1) \\
\text { Error covariance update equation } \\
P(k+1 \mid k+1) \\
=[I-K(k+1) H(k+1, \hat{x}(k+1 \mid k))] P(k+1 \mid k)\end{array}$ \\
\hline Initial condition & \\
\hline$\hat{x}(0 \mid 0), P(0 \mid 0), Q(k), R(k)$ & \\
\hline
\end{tabular}

Figure 2. The STF Process and Sampling Period

In Figure 2,

$$
\begin{aligned}
& F_{x}(k)=\frac{\partial f(x(k), u(k), k)}{\partial \hat{x}(k)}=\left.\frac{\partial f(x(k), u(k), k)}{\partial x}\right|_{x=\hat{x}(k)} \\
& H_{x}(k)=\frac{\partial h(x(k), k)}{\partial \hat{x}(k \mid k-1)}=\frac{\partial h(x(t), t)}{\partial x} \mid \begin{array}{l}
\mid x=\hat{x}(k \mid k-1) \\
\Lambda_{i}(k+1)=\operatorname{diag}\left(\lambda_{i 1}(k+1), \lambda_{i 2}(k+1), \cdots, \lambda_{i n}(k+1)\right.
\end{array} \\
& \lambda_{i}=\left\{\begin{array}{l}
\alpha_{i} \eta(k+1) ; \alpha_{i} \eta(k+1)>1 \\
1 ;
\end{array} \alpha_{i} \eta(k+1) \leq 1\right. \\
& \eta(k+1)=\frac{\operatorname{tr}[N(k+1)]}{\sum_{i=1}^{n} \alpha_{i} M{ }_{i i}(k+1)} \\
& N(k+1)=V_{0}(k+1)-\beta R(k+1)-H(k+1, \hat{x}(k+1 \mid k)) \Gamma(k) Q(k) \Gamma^{\mathrm{T}}(k) H^{\mathrm{T}}(k+1, \hat{x}(k+1 \mid k))
\end{aligned}
$$




$$
\begin{aligned}
& M(k+1)=F(k, u(k), \hat{x}(k \mid k)) P(k \mid k) F^{\mathrm{T}}(k, u(k), \hat{x}(k \mid k)) H^{\mathrm{T}}(k+1, \hat{x}(k+1 \mid k)) H(k+1, \hat{x}(k+1 \mid k)) \\
& =\left(M_{i j}(k+1)\right) \\
& V_{0}(k+1)=\mathrm{E}\left[\gamma(k+1) \gamma^{\mathrm{T}}(k+1)\right] \approx\left\{\begin{array}{l}
\gamma(1) \gamma^{\mathrm{T}}(1), \quad k=0 \\
\frac{\left[\rho V_{0}(k)+\gamma(k+1) \gamma^{\mathrm{T}}(k+1)\right]}{1+\rho}, k \geq 1
\end{array}\right. \\
& \gamma(k+1)=y(k+1)-h(k+1, \hat{x}(k+1 \mid k))
\end{aligned}
$$

In the formula $(5) \sim(10), \alpha_{i} \geq 1, i=1,2, \cdots, n$ are pre-selected coefficient; $\beta \geq 1$ is preselected weakened factor; $\operatorname{tr}[\cdot]$ is matrix trace; $V_{0}(k+1)$ is mean-square error matrix of output sequence; $\gamma(k+1)$ is residual sequence; $0<\rho \leq 1$ is forget factor, $(\rho=0.95)$; $\hat{x}(0 \mid 0)$ and $P(0 \mid 0)$ are initial value.

\subsection{Optimal Estimate of Multi-sensors Linear Combination}

Figure 3 is the block diagram of vehicle state online estimation system based on multisensors linear combination and STF theory. The vehicle state optimal estimation system includes four lateral acceleration sensors, single sensor STF, sensors fault diagnosis, system information fusion and main filter. The information of vehicle lateral acceleration can be observed with the 4 sensors in Figure 5. In order to make the system has more precision, the each sensor information are filtered with the STF. And also, the faults of sensors are checked in time to insure the output information reliable. Then, the accurate vehicle non-linear state estimation $\hat{x}(t)$ after the main filter can be gained by the linearity combination. The vehicle key states estimated value can be used in the vehicle closedloop accurate control.

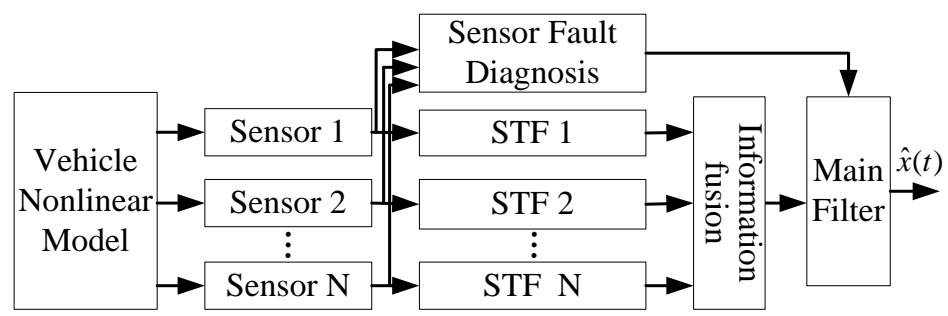

Figure 3. The Block Diagram of Vehicle State Online Estimation System

$\hat{x}_{i}(k \mid k) \quad(i=1,2,3,4)$ are obtained by using STF to each sensor. More precise integrated tracking can be acquired by linear optimization combination with $\hat{x}_{i}(k \mid k)$. As $\hat{x}_{i}(k \mid k)$ is a state variable $x(k)$ of the four unbiased estimate, the estimation error and the error covariance matrix of $\hat{x}_{i}(k \mid k)$ are as following.

$\tilde{x}_{i}(k \mid k)=x(k)-\hat{x}_{i}(k \mid k)$

$P_{i}(k \mid k)=E\left\{\tilde{x}_{i}(k \mid k) \tilde{x}_{i}^{T}(k \mid k)\right\}$

With unbiased estimation of the linear combination of $\hat{x}(k \mid k)$, then

$\hat{x}(k)=\sum_{i=1}^{4} a_{i} \hat{x}_{i}(k \mid k) ; \quad a_{1}+a_{2}+a_{3}+a_{4}=1$

Where, $a_{i}$ is weighted coefficient. The main issues for optimal amalgamation estimate of multi-sensor linear combination are changed into solve the problems of coefficients weighted of $\hat{x}_{i}(k \mid k)$. 
Then, $P(k)$ is covariance matrix of error $\tilde{x}(k)=x(k)-\hat{x}(k)$, for that $\tilde{x}_{i}(k)$ is irrelevant to $\tilde{x}_{j}(k)(i \neq j)$

$P(k)=E\left\{\tilde{x}(k) \tilde{x}^{T}(k)\right\}=\sum_{i=1}^{4} a_{i} P_{i}(k)$

The minimum criterion is established as following:

$J=\operatorname{tr}[P(k)]=\sum_{i=1}^{4} a_{i}^{2} \operatorname{tr}\left[P_{i}(k)\right]$

Then Lagrange multipliers method is used, and auxiliary functions are introduced.

$f=\sum_{i=1}^{N} a_{i}^{2} \operatorname{tr}\left[P_{i}(k)\right]+\lambda\left(\sum_{i=1}^{4} a_{i}-1\right)$

$\frac{\partial f}{\partial a_{i}}=2 a_{i} \operatorname{tr}\left[P_{i}(k)\right]+\lambda=0$. Finally, the weighted coefficient of the optimal amalgamation estimate of multi-sensor linear combination is obtained.

$a_{i}=1 / \operatorname{tr}\left(P_{i}(k)\right) / \sum_{i=1}^{4}\left(1 / \operatorname{tr}\left(P_{i}(k)\right)\right.$

\section{Vehicle Handling Dynamics State Estimation with STF}

\subsection{Vehicle Nonlinear Dynamic Modeling}

The vehicle dynamic model is an important precondition to prove the effectiveness of the control laws. However, it is complex and cannot be described precisely because the system parameters vary with the time and the longitudinal / lateral dynamic have strong coupling effects. In this paper, the 4-DOF vehicle nonlinear dynamic model including longitudinal, lateral, roll and yaw is shown as Figure 4.

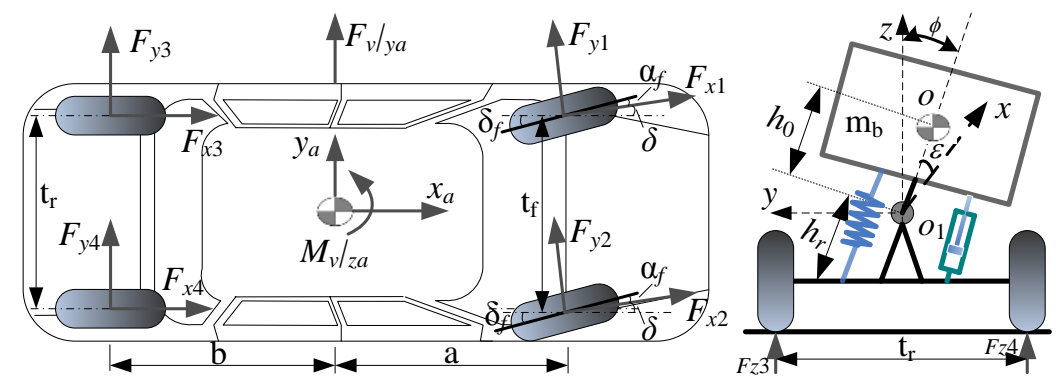

Figure 4. Vehicle Dynamic Model

Supposed the steering angle $(\delta)$ and roll angle $(\varnothing)$ are very small, i.e., $\cos \delta=1, \sin \delta \approx \delta, \sin \phi \approx \phi$. Then, the full vehicle dynamic model can be described with the Newton Second Law, as following. 


$$
\begin{aligned}
& \int m\left(\dot{u}-v r-r \dot{\phi} h_{0} \cos \varepsilon\right)=\left(F_{x 1}+F_{x 2}\right) \cos \delta-\left(F_{y 1}+F_{y 2}\right) \sin \delta+F_{x 3}+F_{x 4} \\
& m\left(\dot{v}+u r+r \dot{\phi} h_{0} \cos \varepsilon\right)=\left(F_{y 1}+F_{y 2}\right) \cos \delta+\left(F_{x 1}+F_{x 2}\right) \sin \delta+F_{y 3}+F_{y 4} \\
& I_{b, z} \dot{r}+\left(I_{b, z} \sin \varepsilon-I_{b, x z} \cos \varepsilon\right) \phi=a \cdot\left[\left(F_{y 1}+F_{y 2}\right) \cos \delta+\left(F_{x 1}+F_{x 2}\right) \sin \delta\right]-b\left(F_{y 3}+F_{y 4}\right) \\
& +\frac{t_{f}}{2}\left[\left(F_{y 1}-F_{y 2}\right) \sin \delta-\left(F_{x 1}-F_{x 2}\right) \cos \delta\right]-\frac{t_{r}}{2}\left(F_{y 3}-F_{y 4}\right) \\
& \left(I_{b, x} \cos \varepsilon-I_{b, x z} \sin \varepsilon\right) \ddot{\phi}-I_{b, x z} \dot{r}-m h_{0}(\dot{v}+r u)=-\left(k_{f} \frac{t_{f}^{2}}{2}+k_{r} \frac{t_{r}^{2}}{2}\right) \phi-\left(c_{f} \frac{t_{f}^{2}}{2}+c_{r} \frac{t_{r}^{2}}{2}\right) \dot{\phi} \\
& +\left(h_{r f}-h_{r}\right) \sum_{i=1}^{2} F_{y i}+\left(h_{r r}-h_{r}\right) \sum_{i=3}^{4} F_{y i}
\end{aligned}
$$

Where, the tires longitudinal and lateral forces $F_{x 1,2}, F_{y 1,2}, F_{x 3,4}, F_{y 3,4}$ are calculated with Magic Formula model. $u$ is vehicle longitudinal velocity; $v$ is vehicle lateral velocity; $r$ is yaw rate; $p$ is roll angular velocity; $\Phi$ is roll angle; $I_{b, x}, I_{b, z}$ are roll and yaw Inertia moment respectively; $I_{b, x z}$ is roll/yaw cross inertia moment; $m$ is full vehicle mass; $m_{b}$ is up-spring mass; $a, b$ are distances from the front and rear axle to the center of mass respectively; $t_{f}, t_{r}$ are front and rear axle wheelbase respectively; $h_{0}$ is the distance from center of mass to roll axle; $h_{r}$ is the high of roll center; $\varepsilon$ is inclined angle of roll axel and longitudinal axle; $h_{r r}$ is the distance from rear axle to roll axle; $k_{f}, k_{r}$ are front and rear suspension stiffness respectively; $k_{s t}$ is the stiffness of front stabilizer bar; $c_{f}, c_{r}$ are front and rear suspension damping; $\delta$ is the rotate angle of front wheel.

\subsection{Vehicle State Estimation Based on STF}

The four lateral accelerometers are the measurable variables, and the sensors are positioned as shown in Figure 5.

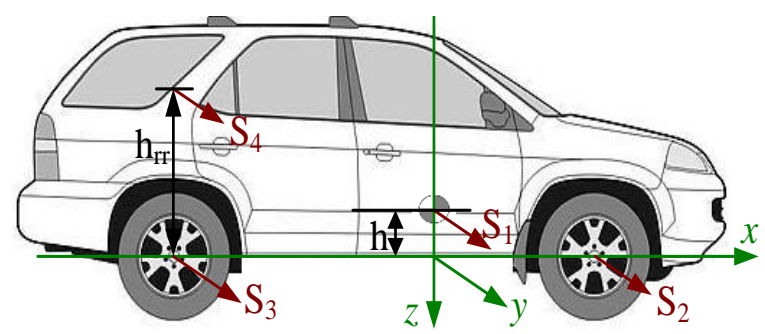

Figure 5. Sensors Placement and Orientation

The model of lateral acceleration sensors are as following.

$\begin{cases}a_{y 1}=\dot{v}+h \dot{p}+r u+\eta_{1} ; & a_{y 2}=\dot{v}+a \dot{r}+r u+\eta_{2} \\ a_{y 3}=\dot{v}-b \dot{r}+r u+\eta_{3} ; & a_{y 4}=\dot{v}+h_{r r} \dot{p}-b \dot{r}+r u+\eta_{4}\end{cases}$

where, $\eta_{i}(i=1,2,3,4)$ are the measure noise of sensors.

According to the vehicle nonlinear dynamics model (1) and the model of sensors, the vehicle nonlinear state equation and observation equation are obtained.

$\left\{\begin{array}{l}\dot{x}(t)=f(x(t), u(t), t)+w(t) \\ y_{i}(t)=h_{i}(x(t), t)+\eta_{i}(t)\end{array}\right.$

Where, the state variable is $x(t)=[u, v, \gamma, p, \phi]^{\mathrm{T}}$; input variable is $u(t)=[\delta]$; observation vector is $y(t)=\left[a_{y 1}, a_{y 2}, a_{y 3}, a_{y 4}\right]^{\mathrm{T}}$ which are obtained by the sensors positioned in vehicle. Random variables $w(t)$ and $\eta_{i}(t)$ are process noise and measured noise 
respectively. These two kinds of noise are independent with each other. They are gaussian white noise with zero mean and their probability distributions are as following.

$$
\begin{aligned}
& E[w(t)]=E\left[\eta_{i}(t)\right]=0 \quad E\left[w(t) \eta_{i}{ }^{\mathrm{T}}(j)\right]=0 \\
& E\left[w(t) w^{\mathrm{T}}(j)\right]=Q \delta_{t j} \quad E\left[\eta_{i}(t) \eta_{i}{ }^{\mathrm{T}}(j)\right]=R \delta_{t j}
\end{aligned}
$$

Supposed the sampling period is $T$. The system continuous state equations and observation equations are dispersed to acquire the state equations and observation equations of discrete systems.

$\left\{\begin{array}{l}\dot{\tilde{x}}(k+1)=f(x(k), u(k), k)+w(k) \\ \tilde{y}_{i}(k)=h_{i}(x(k), k)+\eta_{i}(k)\end{array}\right.$

According to the equation 2) and 3) of formula (19), the estimation model of yaw rate can be gained as following.

$\dot{\gamma}=\frac{\left(a_{y 2}-a_{y 3}\right)+\left(\eta_{2}-\eta_{3}\right)}{a+b}$

The same argument, according to the equation 3) and 4) of formula (19), the estimation model of vehicle roll angle also can be gained.

According to the formula (18), (19) and (22), the state equation and observation equation of vehicle are nonlinear. Assumption that the longitudinal velocity $u$ and the wheel speeds are invariable, the state variables change into $x(t)=[v, \gamma, p, \phi]^{\mathrm{T}}$.

A supposed can be made that filtering estimate $\hat{x}(k)$ of state $x(k)$ has obtained in the $k_{t h}$ time, around the filtering estimate $\hat{x}(k)$, the nonlinear function $f(x(k), u(k), k)$ is evolved into Taylor series. Ignoring the second order and above, the vehicle state equation is almost linearization as following.

$\dot{\tilde{x}}(k+1) \approx f(\hat{x}(k), u(k), k)+F_{x}(k)[x(k)-\hat{x}(k)]+w(k)$

The observation equation is linearization as following.

$\tilde{y}_{i}(k) \approx h_{i}(\hat{x}(k \mid k-1), k)+H_{x i}(k)[x(k)-\hat{x}(k \mid k-1)]+\eta_{i}(k)$

According to the formula (2) and (3), $F_{x}(k)$ and $H_{x i}(k)$ are the partial derivations aimed state $x(k)$ of nonlinear function $f(x(k), u(k), k)$ and $h_{i}(x(k), k)$ respectively.

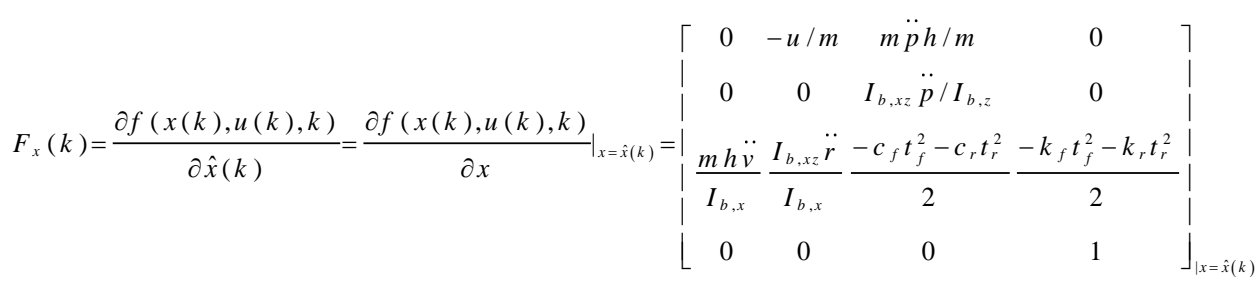

$$
\begin{aligned}
& \left.H_{x}(k)=\frac{\partial h(x(k), k)}{\partial \hat{x}(k \mid k-1)}=\left.\frac{\partial h(x(t), t)}{\partial x}\right|_{x=\hat{x}(k \mid k-1)}=\mid \begin{array}{cccc}
\ddot{v} & u & h \ddot{p} & 0 \\
\ddot{v} & a \ddot{r}+u & 0 & 0 \\
\ddot{v} & -b \ddot{r}+u & 0 & 0 \\
\ddot{v} & -b \ddot{r}+u & h_{r r} \ddot{p} & 0
\end{array}\right]_{\mid x=\hat{x}(k \mid k-1)}
\end{aligned}
$$

\section{Simulation and Analysis}

Combined with the state variables of vehicle estimated by using STF and the multisensor linear combination optimal estimate theory, an assumption can be made that the system initial state are $x(0)=0, \mathrm{P}(0 \mid 0)=\operatorname{diag}[1,1,1,1], \hat{x}(0 \mid 0)=0$, the initial error matrix are 
$Q_{i}=I_{4 \times 4}, R_{i}=0.001$ and $\beta=0.95$. Sampling time $T=0.05 \mathrm{~s}$. The vehicle parameters are in Table 1.

Table 1. Vehicle Parameter

\begin{tabular}{cc||cc}
\hline parameter & numerical value & parameter & numerical value \\
\hline$m$ & $864.7 \mathrm{~kg}$ & $h_{0}$ & $0.032 \mathrm{~m}$ \\
$m_{s}$ & 745.2 & $h_{r f}$ & $0.2 \mathrm{~m}$ \\
$m_{u}$ & $119.5 \mathrm{~kg}$ & $h_{r r}$ & $0.5 \mathrm{~m}$ \\
$a$ & $1.1161 \mathrm{~m}$ & $\varepsilon$ & $6.8428 \mathrm{deg}$ \\
$b$ & $1.2319 \mathrm{~m}$ & $s_{f}$ & $1.2 \mathrm{~m}$ \\
$\mathrm{~h}$ & $0.468 \mathrm{~m}$ & $s_{r}$ & $1.2 \mathrm{~m}$ \\
$t_{f}$ & $1.56 \mathrm{~m}$ & $K_{f}$ & $6000 \mathrm{~N} / \mathrm{m}$ \\
$t_{r}$ & $1.54 \mathrm{~m}$ & $K_{r}$ & $6500 \mathrm{~N} / \mathrm{m}$ \\
$I_{x}$ & $375.2 \mathrm{kgm}^{2}$ & $K_{s t}$ & $22918 \mathrm{Nm} / \mathrm{rad}$ \\
$I_{y}$ & $768.8 \mathrm{kgm}^{2}$ & $B_{f}$ & $4200 \mathrm{Nm} / \mathrm{s}$ \\
$I_{z}$ & $806.4 \mathrm{kgm}^{2}$ & $B_{r}$ & $4200 \mathrm{Nm} / \mathrm{s}$ \\
\hline
\end{tabular}

Supposed the longitudinal velocity is steady and $u=20 \mathrm{~m} / \mathrm{s}$. The curve of continuous input signal of steering angle and lateral acceleration signals are showed in Figure 6.
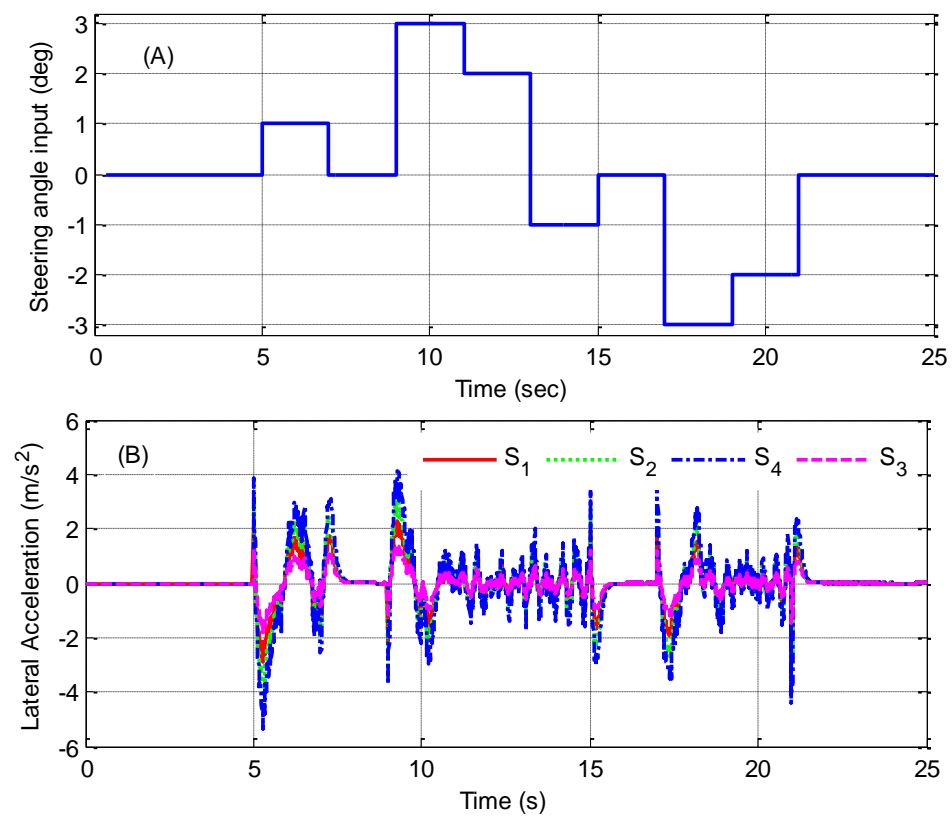

Figure 6. The Signals of Steering Angle and Lateral Acceleration

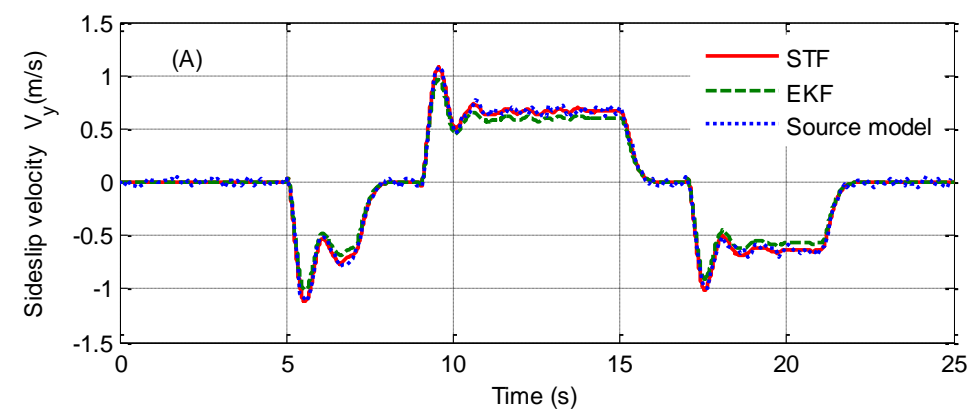



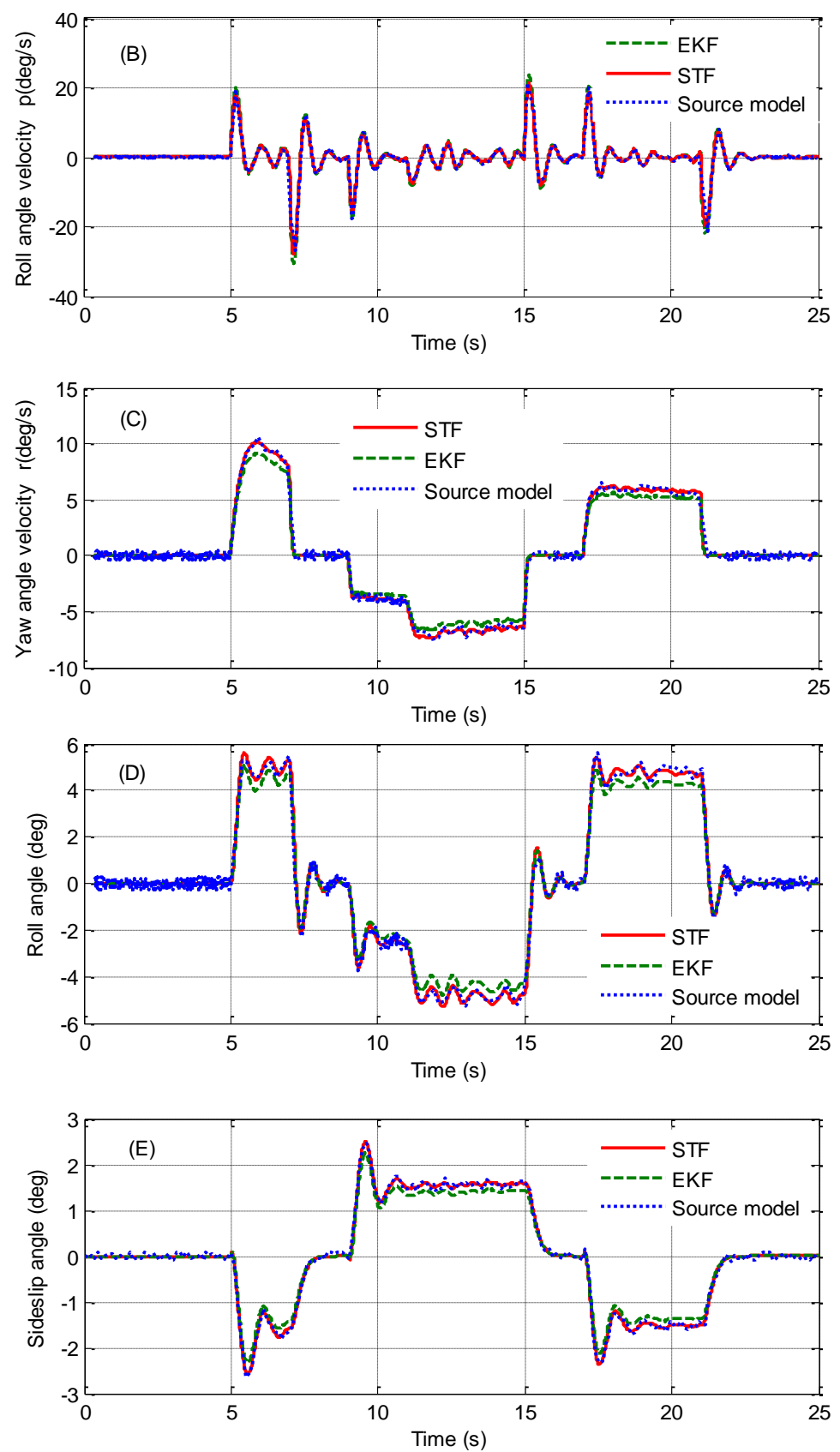

Figure 7. Performance Comparison between EKF and STF

Figure 7 (A, B, C, D and E) are the estimated results of the vehicle key state such as lateral velocity, roll angular velocity, yaw angular velocity, roll angle and vehicle body side slip angle based on STF and the EKF. Figure 6 shows that filters estimate roll rate, yaw rate and roll angle are extremely well. Besides, the errors can be controlled in small bound. Ultimately, the stability of the vehicle speed tends to zero. This phenomenon is reasonable because the non-linearity of the vehicle model in $p, \gamma$ and $\theta$ states are relatively insensitive to the tires non-linearity, which has the biggest non-linearity effect in the vehicle model. From equation (18), it can be shown that the roll dynamics are strongly affected by spring-damper forces of the suspension, which are linear in the model. Also, it can be shown that the yaw dynamics are strongly affected by lateral tires forces. However, since the overall yaw moment is caused by the subtraction between tires lateral moment of front wheels and that of rear wheels, the overall non-linearity affected by tires 
lateral forces is reduced. Yaw and roll modes are also well accommodated within the sensor feedback. So non-linearity errors are rapidly diminished.

The biggest non-linearity effect occurs in side-slip velocity dynamics. Equation (18) also shows that side-slip is caused by the addition of all four lateral tires forces, so this is strongly influenced by tires non-linearity. When the lateral acceleration change acutely (as shown in Figure 6, the time such as $5-7 \mathrm{sec}, 9-15 \mathrm{sec}$ ), there is a certain error in the state estimation method. But the SFT estimated accuracy is slightly higher than the EKF. The reason is that the initial error makes sub-optimal fading factors have a regulatory role to amend the state estimation error. So, the state estimate is more accurate than the EKF.

\section{Conclusions}

According to the vehicles lateral acceleration and steering wheel sensor information easily measured, combining with the vehicle dynamics model, the system equations of state and measurement equation for vehicle nonlinear state estimation are built. With the EKF and STF technology, the key states (such as vehicle lateral velocity, yaw rate and side slip angle) are estimated. The simulation results show that the STF and EKF filtering method based on multi-sensors data fusion have more effective result, and the estimated error is small. When the lateral acceleration become stable, state estimation error can quickly converge to zero and come into stable state.

Multi-sensors data fusion and STF theory can improve the reliability of the vehicle state estimation system and the accuracy of estimates. The computer simulation results show that this method is very effective for vehicle state estimation.

\section{Acknowledgements}

Authors would like to thank anonymous reviewers and the editor for their valuable comments and the National Natural Science Foundation of China (No.51278514) and the Natural Science Foundation of Chongqing (No.cstc2014jcyjA6007) and the Special Fund of Chongqing Education Commission (KJ120415) for their sponsorship.

\section{References}

[1] D. Piyabongkarnl and R. Rajamani, "Development and experimental evaluation of a slip angle estimator for vehicle stability control", Proceedings of the American Control Conference, (2006); Minnesota, USA.

[2] K. Nam, S. Oh and H. Fujimoto, "Vehicle state estimation for advanced vehicle motion control using novel lateral tire force sensors", Proceedings of the American Control Conference, (2011); USA.

[3] H. Cherouat, "Vehicle velocity, side slip angles and yaw rate estimation", IEEE ISIE, (2005); Dubrovnik, Croatia.

[4] H. Lee, "Reliability indexed sensor fusion and its application to vehicle velocity estimation", Journal of Dynamic Systems, Measurement, and Control, vol.128, no.2, (2006), pp.236-243.

[5] W. A. Thomas and K. J. Burnham, "Dual extended kalman filter for vehicle state and parameter estimation", Vehicle System Dynamics, vol.44, no.2, (2006), pp.153-171.

[6] W. A. Thomas and K. J. Burnham, "Simplified extended kalman filter for automotive state estimation", International Journal of Modeling", Identification and Control, vol.3, no.3, (2008), pp.201-211.

[7] M. Satria and M. C. Best, "Comparison between Kalman Filter and Robust Filter for vehicle handling dynamics state estimation", SAE, (2002).

[8] H. Lee, "Reliability indexed sensor fusion and its application to vehicle velocity estimation", Journal of Dynamic Systems, Measurement and Control, vol.128, no.2, (2006), pp.236-243.

[9] A. Rezaeian, R. Zarringhalam, S. Fallah and W. Melek, "Cascaded Dual Extended Kalman Filter for combined vehicle state estimation and parameter identification", SAE, (2013).

[10] G. Agamennoni, S. Worrall and J. Ward, "Robust non-linear smoothing for vehicle state estimation", Proceedings of the IEEE Intelligent Vehicles Symposium (IV), (2013); Gold Coast, Australia.

[11] A. Katriniok, J. P. Maschuw and F. Christen, "Optimal vehicle dynamics control for combined longitudinal and lateral autonomous vehicle guidance", Proceedings of the European Control Conference, (2013); Zurich, Switzerland. 
[12] J. Zhao, R. Zhao and F. He, "Vehicle lateral states estimation using Kalman-Bucy Filter", Proceedings of the FISITA World Automotive Congress Lecture Notes in Electrical Engineering, (2013).

[13] D. H. Zhou, "Introduction to adaptive control of non-linear systems", Tsinghua University Press and Springer Press, (2002).

[14] Z.L. Deng, "Optimal filter theory and its application”, Harbin Institute Technology Press, (2000).

\section{Authors}

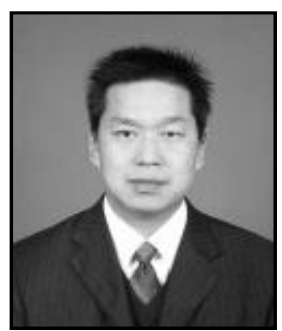

Shu-en Zhao, he received his B.S. from Chang'an Univ. in 1996, M.S. and Ph.D degrees in Vehicle Engineering from Chongqing Univ. in 2005 and 2010, respectively. Currently, he is an Associate Professor in Chongqing JiaoTong Univ., China. His interests include vehicle dynamic and control, vehicle lightweight design and vehicle $\mathrm{NVH}$.

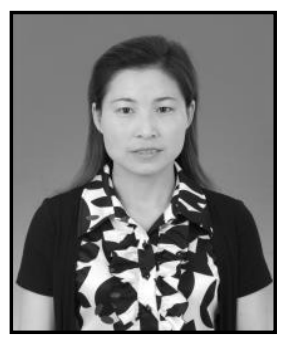

Yu-ling Li, she received his B.S. from Chang'an Univ. in 1996, M.S. degree in Mechanical Engineering from Chongqing Univ. in 2008. Currently, she is an Associate Professor in Chongqing JiaoTong Univ., China. Her interests include vehicle dynamic and control and advance manufacturing technology. 
International Journal of Control and Automation Vol. 8, No. 9 (2015) 